\title{
Divergent Integrals, Residues of Dolbeault Forms, and Asymptotic Riemann Mappings
}

\section{Giovanni Felder ${ }^{1, *}$ and David Kazhdan ${ }^{2}$}

${ }^{1}$ Department of Mathematics, ETH Zurich, 8092 Zurich, Switzerland and

${ }^{2}$ Einstein Institute of Mathematics, The Hebrew University of

Jerusalem, Jerusalem 91904, Israel

*Correspondence to be sent to: e-mail: felder@math.ethz.ch

We describe the asymptotic behaviour and the dependence on the regularization of logarithmically divergent integrals of products of meromorphic and antimeromorphic forms on complex manifolds. Our formula is expressed in terms of residues of Dolbeault forms, a notion introduced in this paper. The proof is based on a result on the asymptotic behaviour of Riemann mappings of small domains.

\section{Introduction}

Let $X$ be a compact complex manifold of dimension $n$ and $D \subset X$ a smooth hypersurface. We are interested in regularizations of divergent integrals of the form

$$
\frac{1}{2 \pi \mathrm{i}} \int_{X} \alpha \wedge \bar{\beta}, \quad \alpha \in \Gamma\left(X, \Omega_{X}^{n}(m D)\right), \quad \beta \in \Gamma\left(X, \Omega_{X}^{n}(D)\right) .
$$

Here $\Gamma\left(X, \Omega_{X}^{p}(m D)\right)$ denotes the space of meromorphic $p$-forms on $X$ whose poles are on $D$ and are of order at most $m$. Integrals of this type arise in perturbative superstring theory, see [6], particularly Section 7.6: the terms in the perturbation series for superstring amplitudes are conditionally convergent improper integrals of products of holomorphic times antiholomorphic differential forms on moduli spaces of supercurves. They thus

Received March 25, 2016; Revised July 2, 2016; Accepted August 8, 2016

(C) The Author(s) 2016. Published by Oxford University Press. All rights reserved. For permissions, please e-mail: journals.permission@oup.com. 
depends on the way one takes the limit and it is important to understand this dependence. We consider here the basic case of an integral of this kind, with only one divisor component.

The way to make sense of integrals of the form above is to introduce a regularization and study the asymptotic behaviour as the regularization is removed. Specifically the regularization consists of replacing the integral over $X$ by the integral over the complement of a suitable family of tubular neighbourhoods $N_{\epsilon}(D)$ of $D$ shrinking to zero size as the regularization parameter $\epsilon>0$ tends to zero. It turns out that the integral behaves as $I_{0} \log \epsilon+I_{1}+O(\epsilon)$ as $\epsilon \rightarrow 0$, with $I_{0}$ independent of the choice of family of neighbourhoods. In applications to superstring theory the integral is "finite" in the sense that $I_{0}$ actually vanishes, so that one can define the integral as $I_{1}$. However $I_{1}$ depends on the choice of regularization and it is important to understand this dependence. The aim of this article is to describe the divergent term and dependence on the choice of regularization, generalizing calculations of [6], where the case $m=1$ is discussed. More precisely, we consider regularizations defined by a cut-off function $\lambda$, a smooth positive function on $X \backslash D$ such that $\lambda /|f|$ locally extends smoothly to a positive function on $X$ for any local equation $f=0$ of $D$ such that $\left.d f\right|_{D} \neq 0$. For example, one can take $\lambda$ around $D$ to be the geodesic distance to $D$ for some Hermitian metric on $X$ and extend it to a positive smooth function on $X \backslash D$. Then for small $\epsilon>0, N_{\epsilon}(D)=\{x \in X: \lambda(x)<\epsilon\}$ is a tubular neighbourhood of $D$ and we define the regularized integral as the integral over its complement.

The first result is that the divergent part can be written in terms of residues:

$$
\frac{1}{2 \pi \mathrm{i}} \int_{\lambda \geq \epsilon} \alpha \wedge \bar{\beta}=2(-1)^{n-1} \log \epsilon \int_{D} \operatorname{Res} \alpha \wedge \overline{\operatorname{Res} \beta}+I_{1}(\lambda)+O(\epsilon), \quad(\epsilon \rightarrow 0) .
$$

Here Res is the Leray residue, sending de Rham cohomology classes on $X \backslash D$ to de Rham cohomology classes on $D$. For meromorphic closed forms with first order poles on $D$, such as $\beta$, it reduces to the Poincaré residue, which is defined as a holomorphic differential form on $D$, not just a cohomology class. It follows in particular that the coefficient of $\log \epsilon$ only depends on the de Rham cohomology classes of the closed forms $\alpha$ and $\beta$.

To describe the dependence of the finite part $I_{1}(\lambda)$ on the choice of $\lambda$ we notice that any two cut-off functions differ by multiplication by $\exp \varphi$ for some smooth real-valued function $\varphi$. We find:

$$
I_{1}\left(e^{\varphi} \lambda\right)=I_{1}(\lambda)-2(-1)^{n-1} \int_{D} \operatorname{Res}_{\partial}(\varphi \alpha) \wedge \overline{\operatorname{Res} \beta}
$$


see Theorem 3.2. Here $\operatorname{Res}_{\partial}$ is the residue of a Dolbeault form, a notion we introduce in this article. It maps smooth forms $\omega$ of type $(n, 0)$ on $X \backslash D$ with poles on $D$ to classes of forms on $D$ of type $(n-1,0)$ on $D$ modulo the image of $\partial$ (it is more convenient in this setting to consider $\partial$ as the Dolbeault differential; it is related to the usual convention by complex conjugation).

A remarkable feature of (2) is that the variation of the integral is linear in $\varphi$ and is independent of $\lambda$.

Our formulas are a special case of a more general formula where one takes $\alpha$ to be a not necessarily closed $C^{\infty}$ form of type $(n, 0)$ on $X \backslash D$ and whose support has compact closure in $X$ (we don't need $X$ to be compact in this case). The formulas (1) and (2) hold except that one has to replace $\operatorname{Res} \alpha$ in (1) by $\operatorname{Res}_{\partial} \alpha$. This more general formula can be checked locally via a partition of unity and the formula for meromorphic $\alpha$ follows as a corollary.

The proof of these statements is based on a result on the asymptotic behaviour of Riemann mappings for small domains, which might be of independent interest. Let $X=\mathbb{C}$ and $D=\{0\}$ and let $\lambda$ be a cut-off function such that $\lambda^{2}$ is real analytic. Then, for small $\epsilon>0, \lambda(z)<\epsilon$ defines a simply connected domain containing the origin, so, by the Riemann mapping theorem, there is a unique biholomorphic map $z \mapsto W=f_{\epsilon}(z)$ on to the disk $|w|<\epsilon$ sending 0 to 0 and such that $f_{\epsilon}^{\prime}(0)>0$. We show (see Theorem 4.1) that the family of maps $f_{\epsilon}(z)$ converges as $\epsilon \rightarrow 0$ to a univalent map defined in a neighbourhood of the origin and is in fact the restriction of a holomorphic function of two variables $\epsilon, z$ defined on a polydisk around $(0,0)$. In Theorem 4.2 we give an explicit formula for the limiting map.

This construction is used to prove (1), (2) by first reducing to a local calculation and then reducing to the case where $\lambda=|f|$ for a local equation $f=0$ of $D$.

The article is organized as follows. In Section 2 we introduce a notion of residue for $C^{\infty}$ differential forms of type $(p, 0)$ on a complex manifold with poles on a smooth hypersurface. The divergent part of the integral and the dependence on the cut-off of the finite part are expressed in terms of this residue in Section 3. Our result on the asymptotic behaviour of Riemann mappings for small domains is proved in Section 4. Section 5 contains the proof of the formulas for divergent integrals presented in Section 3.

\section{Residues of Dolbeault Forms}

Let $X$ be an $n$-dimensional complex manifold with sheaf $\mathcal{O}_{X}$ of germs of holomorphic functions and let $D \subset X$ be a smooth hypersurface. Thus every point of $D$ has an open 
neighbourhood $U$ so that the ideal of $\mathcal{O}_{X}$ of functions vanishing on $D \cap U$ is generated by a function $f \in \mathcal{O}_{X}(U)$. We call $f$ a local equation for $D$. Any two local equations differ by multiplication by a nowhere vanishing holomorphic function.

\subsection{Residues of smooth differential forms}

A $C^{\infty}$ differential form $\alpha$ on $X \backslash D$ is said to have a pole of order $\leq m$ if around every point of $D, f^{m} \alpha$ extends smoothly to $X$ for a local equation $f$ for $D$. If $\alpha$ is closed and has a pole of order $\leq 1$ then locally $\alpha=\mathrm{d} f / f \wedge \sigma+\tau$ for some smooth forms $\sigma, \tau$. Then $\left.\sigma\right|_{D}$ is closed, globally defined, and independent of choices; it is called Poincaré residue Res $\alpha$, see [5, p. 83]. If $\alpha$ is a holomorphic closed form then $\operatorname{Res} \alpha$ is also holomorphic. The Leray residue of a closed differential form $\alpha$ on $X \backslash D$ is a de Rham cohomology class on $D$. It is defined to be the Poincaré residue for forms with first order pole and in general it is the residue of any form with first order pole in the de Rham cohomology class of $\alpha$, [5, Theorem 1]. These notions were further developed in [1,2]. In this article we develop part of the theory for the (conjugate) Dolbeault complex replacing the de Rham complex.

\subsection{Dolbeault forms}

Let $C_{X}^{\infty}$ be the sheaf of germs of smooth functions on $X$. Let $\mathcal{A}_{X}^{r}=\oplus_{p+q=r} \mathcal{A}_{X}^{p, q}$ be the decomposition of the $C_{X}^{\infty}$-module of smooth differential $r$-forms into forms of type $(p, q)$. The de Rham differential on $\mathcal{A}_{X}^{\bullet}=\oplus_{r=0}^{2 n} \mathcal{A}_{X}^{r}$ decomposes as $d=\partial+\bar{\partial}$ with

$$
\partial\left(\mathcal{A}_{X}^{p, q}\right) \subset \mathcal{A}_{X}^{p+1, q}, \quad \bar{\partial}\left(\mathcal{A}_{X}^{p, q}\right) \subset \mathcal{A}_{X}^{p, q+1} .
$$

Let $\mathcal{A}_{X}^{p, q}(* D)$ be the sheaf of smooth $(p, q)$-forms $\alpha$ on $X \backslash D$ so that $f^{m} \alpha$ extends to $X$ for some $m$ and local equation $f$ for $D$.

Similarly, let $\mathcal{A}_{X}^{p, q}\langle D\rangle$ be the sheaf of smooth logarithmic forms. By definition, sections of $\mathcal{A}_{X}^{p, q}\langle D\rangle$ on an open set $U \subset X$ are smooth $(p, q)$ forms $\alpha$ on $U \backslash(U \cap D)$ such that for any local equation $f$, both $f \alpha$ and $\mathrm{d} f \wedge \alpha$ are regular on $U$. Both $\mathcal{A}_{X}^{p, q}(* D)$ and $\mathcal{A}_{X}^{p, q}\langle D\rangle$ are double complexes with differentials $\partial, \bar{\partial}$. The de Rham sheaves of meromorphic forms $\Omega_{X}^{\bullet}(* D), \Omega_{X}^{\bullet}\langle D\rangle$ are subcomplexes of $\left(\mathcal{A}_{X}^{\bullet, 0}(* D), \partial\right),\left(\mathcal{A}_{X}^{\bullet, 0}\langle D\rangle, \partial\right)$, respectively.

Proposition 2.1. The inclusion map

$$
\left(\mathcal{A}_{X}^{\bullet, 0}\langle D\rangle, \partial\right) \rightarrow\left(\mathcal{A}_{X}^{\bullet, 0}(* D), \partial\right)
$$

is a quasi-isomorphism. The same holds if we take sections with compact support. 
Proof. Let $F_{j}^{\bullet}$ be the subsheaf of $\mathcal{A}_{X}^{\bullet, 0}(* D)$ consisting of sections $\alpha$ such that $f^{j+1} \alpha$ and $f^{j} d f \wedge \alpha$ extend smoothly to $D$ for any local equation $f$. These subsheaves form an increasing filtration

$$
\mathcal{A}_{X}^{\bullet, 0}\langle D\rangle=F_{0}^{\bullet} \subset F_{1}^{\bullet} \subset \cdots \subset \mathcal{A}_{X}^{\bullet, 0}(* D)=\cup_{j} F_{j}
$$

of subcomplexes of $\overline{\mathcal{O}}_{X}$-modules. Locally, the associated graded $F_{j} / F_{j-1}$ consists of classes of sections of the form

$$
\alpha=\frac{\mathrm{d} f}{f^{j+1}} \wedge \sigma+\frac{1}{f^{j}} \tau,
$$

where $\sigma$ and $\tau$ are defined modulo the ideal generated by $f$ and $\mathrm{d} f$. The differential sends $(\sigma, \tau)$ to $(-\partial \sigma-j \tau, \partial \tau)$. For $j \geq 1$, this implies that if $\alpha$ is a closed form then $\tau \equiv-\partial \sigma / j$ $\bmod f, \mathrm{~d} f$ and thus

$$
\alpha=\partial\left(-\frac{1}{j f^{j}} \sigma\right)
$$

in $F_{j} / F_{j-1}$. Thus the cohomology of the associated graded is trivial except for $j=0$, where $F_{0} / F_{-1}=F_{0}$ is the subcomplex of logarithmic forms. The same argument works for the subcomplex of sections with compact support.

Proposition 2.2. The Poincaré residue map on $(n, 0)$ forms uniquely extends to a $C_{X}^{\infty}$ linear map of complexes of $\overline{\mathcal{O}}_{X}$-modules

$$
\operatorname{Res}:\left(\mathcal{A}_{X}^{\bullet, 0}\langle D\rangle / \mathcal{A}_{X}^{\bullet, 0}, \partial\right) \rightarrow\left(i_{*} \mathcal{A}_{D}^{\bullet, 0}[-1], \partial\right)
$$

It restricts to a $C_{X}^{\infty}$-linear map of complexes of $\overline{\mathcal{O}}_{X}$-modules

$$
\operatorname{Res}:\left({ }^{c} \mathcal{A}_{X}^{\bullet, 0}\langle D\rangle /{ }^{c} \mathcal{A}_{X}^{\bullet, 0}, \partial\right) \rightarrow\left(i_{*}{ }^{c} \mathcal{A}_{D}^{\bullet, 0}[-1], \partial\right)
$$

on the subcomplex of forms with compact support.

Proof. Logarithmic forms are locally of the form $\alpha=\mathrm{d} f / f \wedge \sigma \bmod \mathcal{A}_{X}^{\bullet, 0}$ where $\sigma \in \mathcal{A}_{X}^{\bullet, 0}$ and $f$ is a holomorphic function generating the ideal of $D$. We define the residue as $\operatorname{Res} \alpha=\left.\sigma\right|_{D}$. As in the classical case the residue is independent of the choice of $f$ and $\sigma$. By construction it is a map of $C_{X}^{\infty}$-modules and, since $\partial \alpha=-\mathrm{d} f / f \wedge \partial \sigma$, it commutes with the differential (taking into account that the shift functor [-1] involves a change of sign of the differential). It is clear that $\mathcal{A}_{X}^{\bullet, 0}$ lies in the kernel of the residue map. This 
proves existence. As for uniqueness, let $z_{1}, \ldots, z_{n}$ be local coordinates on an open set $U$ such that $D \cap U$ is defined by $z_{1}=0 . \mathcal{A}_{X}^{\bullet, 0}\langle D\rangle(U)$ is an algebra over $C_{X}^{\infty}(U)$ generated by $\mathrm{d} z_{1} / z_{1}, \mathrm{~d} z_{2}, \ldots \mathrm{d} z_{n}$ for which the residue is the classical Poincaré residue and uniqueness follows from $C_{X}^{\infty}$-linearity. It is clear that the residue maps forms with compact support to forms with compact support.

Combining Propositions 2.1 with 2.2 we get maps of complexes (with differential $\partial$ )

$$
\mathcal{A}_{X}^{\bullet, 0}(* D) \longleftarrow \mathcal{A}_{X}^{\bullet, 0}\langle D\rangle \stackrel{\text { Res }}{\longrightarrow} i_{*} \mathcal{A}_{D}^{\bullet, 0}[-1]
$$

where the first arrow is a quasi-isomorphism.

Since these sheaves are fine and thus acyclic for the global section functor, we obtain morphisms of complexes

$$
\Gamma\left(X, \mathcal{A}_{X}^{\bullet, 0}(* D)\right) \longleftarrow \Gamma\left(X, \mathcal{A}_{X}^{\bullet, 0}\langle D\rangle\right) \stackrel{\operatorname{Res}}{\longrightarrow} \Gamma\left(D, \mathcal{A}_{D}^{\bullet, 0}[-1]\right)
$$

and the first arrow is still a quasi-isomorphism. Passing to cohomology we obtain a map

$$
\operatorname{Res}_{\partial}: H^{\bullet}\left(\Gamma\left(X, \mathcal{A}_{X}^{\bullet, 0}(* D)\right), \partial\right) \rightarrow H^{\bullet}\left(\Gamma\left(D, \mathcal{A}_{D}^{\bullet, 0}\right), \partial\right)[-1]
$$

Note that since $\left(\mathcal{A}_{D}^{\bullet, 0}, \partial\right)$ is, by Dolbeault's theorem, a resolution of the sheaf $\overline{\mathcal{O}}_{D}$ of antiholomorphic functions and is acyclic for the functor of global sections, we have:

$$
H^{\bullet}\left(\Gamma\left(D, \mathcal{A}_{D}^{\bullet, 0}\right), \partial\right) \cong H^{\bullet}\left(D, \overline{\mathcal{O}}_{D}\right)
$$

We are mostly concerned with the case of top forms $\mathcal{A}_{X}^{n, 0}(* D)$, which are automatically d-closed.

Definition 2.3. The residue of top Dolbeault forms Res $_{\partial}$ is the composition

$$
\operatorname{Res}_{\partial}: \Gamma\left(X, \mathcal{A}_{X}^{n, 0}(* D)\right) \rightarrow \Gamma\left(D, \mathcal{A}_{D}^{n-1,0}\right) / \operatorname{Im}(\partial) \cong H^{n-1}\left(D, \overline{\mathcal{O}}_{D}\right)
$$

By construction the residue has the following properties:

\section{Proposition 2.4.}

(i) $\operatorname{Res}_{\partial}(\partial \gamma)=0$ for all $\gamma \in \Gamma\left(X, \mathcal{A}_{X}^{n-1,0}(* D)\right)$.

(ii) $\operatorname{Res}_{\partial}$ vanishes on forms extending to smooth forms on $X$. 
(iii) The support of $\operatorname{Res}_{\partial}(\alpha)$ is contained in $D \cap \operatorname{supp}(\alpha)$.

(iv) $\operatorname{Res}_{\partial}$ coincides with the Poincaré residue on $(n, 0)$-forms with simple pole on $D$.

Example 2.5. Let $n=1, D=\{p\}$ a point, $z=x+$ iy a coordinate vanishing at $p$ and $m \geq 1$. Then a local section $\alpha \in \mathcal{A}_{X}^{1,0}(m D)$ has the form $g(x, y) \mathrm{d} z / z^{m}$ for some smooth function $g$ defined in a neighbourhood of $(0,0)$. Then

$$
\operatorname{Res}_{\partial} \alpha=\frac{1}{(m-1) !} \partial_{z}^{m-1} g(0,0), \quad \partial_{z}=\frac{1}{2}\left(\partial_{X}-\mathrm{i} \partial_{Y}\right) .
$$

Indeed, this holds by definition for $m=1$. The general case follows by induction from

$$
g \frac{\mathrm{d} z}{z^{j+1}}=\partial_{z} g \frac{\mathrm{d} z}{j z^{j}}-\partial_{z}\left(\frac{g}{j z^{j}}\right) \mathrm{d} z \equiv \partial_{z} g \frac{\mathrm{d} z}{j z^{j}} \bmod \operatorname{Im} \partial, \quad j \geq 1 .
$$

\subsection{Comparison with the Leray residue}

There is a canonical map

$$
\psi: H^{p}(D, \mathbb{C}) \rightarrow H^{p}\left(\Gamma\left(D, \mathcal{A}_{D}^{\bullet, 0}\right), \partial\right)
$$

from the de Rham cohomology: it sends the class of a closed $p$-form $\alpha$ with Hodge decomposition

$$
\alpha=\alpha^{p, 0}+\alpha^{p-1,1}+\cdots+\alpha^{0, p}
$$

to the class of its top component $\alpha^{p, 0}$.

Proposition 2.6. $\operatorname{Res}_{\partial}(\alpha)=\psi(\operatorname{Res} \alpha)$ for all $\alpha \in \Gamma\left(X, \Omega_{X}^{n}(* D)\right)$.

Proof. If $\alpha$ is a differential form on $X \backslash D$ then its de Rham cohomology class contains a form $\tilde{\alpha}$ with first order pole and the residue is the residue of $\tilde{\alpha}$. Now suppose that $\alpha$ is of type $(n, 0)$ with pole on $D$. Then

$$
\alpha=\tilde{\alpha}+d \gamma=\tilde{\alpha}+\partial \gamma+\bar{\partial} \gamma \equiv \tilde{\alpha}+\partial \gamma \quad \bmod \bar{F}^{1} \mathcal{A}^{\bullet}(* D),
$$

where $\bar{F}^{1} \mathcal{A}^{p}(* D)=\oplus_{q \geq 1} \mathcal{A}^{p-q, q}(* D)$. Then Res $\alpha=\left.\sigma\right|_{D}$, where $\tilde{\alpha}=\mathrm{d} f / f \wedge \sigma+\tau$. On the other hand if we denote by $\alpha_{p}$ the $(p, 0)$ part of a $p$-form $\alpha$,

$$
\alpha=\tilde{\alpha}_{n}+\partial \gamma_{n-1}
$$

Then $\alpha_{n}=\mathrm{d} f / f \wedge \sigma_{n-1}$ and by definition $\operatorname{Res}_{\partial} \alpha=\left.\sigma_{n-1}\right|_{D}=(\operatorname{Res} \alpha)_{n-1}$. 


\section{Divergent Integrals}

The divergent integrals we are interested in are regularizations of integrals of the form $\int_{X} \alpha \wedge \bar{\beta}$ where $\alpha, \beta$ are meromorphic differential $n$-forms on $X$ with poles on $D$ and $\beta$ has a simple pole. The regularization consists on integrating on the complement of a small tubular neighbourhood of radius $\epsilon$ of $D$ and subtracting the divergent term as $\epsilon \rightarrow 0$. The result depends on the regularization and we want to describe this dependence.

Definition 3.1. A cut-off function is a continuous function on $X$ with values in $[0, \infty)$ so that

(i) $\lambda$ is smooth and positive on $X \backslash D$.

(ii) For any local equation $f: U \rightarrow \mathbb{C}$ for $D, \lambda /|f|$ extends to a smooth positive function on $U$.

By a local equation for $D$ we mean a holomorphic function $f$ defined on an open subset $U$ of $X$ such that $U \cap D=f^{-1}(0)$ and $\mathrm{d} f \neq 0$ on $U \cap D$. Cut-off functions exist as they can be constructed by patching local functions $\lambda(x)=|f(x)|$ using a partition of unity. They form a torsor over positive functions, since the ratio of two cut-off functions is a smooth positive function on $X$.

Another interpretation, indicated by the referee, is that cut-off functions are the same as Hermitian metrics on the line bundle $O(D)$ associated with $D$ : if \|\| is such a metric and $s_{D}$ is the canonical section of $O(D)$ (it vanishes exactly on $D$ ), then $\lambda=\left\|s_{D}\right\|$ is a cut-off function.

Theorem 3.2. Let $D \subset X$ be a smooth hypersurface in a compact complex manifold. Let $\lambda \in C^{\infty}(X)$ be a cut-off function and $m \in\{0,1,2, \ldots\}$. Then for any $\alpha \in \Gamma\left(X, \Omega_{X}(m D)\right)$, $\beta \in \Gamma\left(X, \Omega_{X}(D)\right)$,

$$
\frac{1}{2 \pi \mathrm{i}} \int_{\lambda \geq \epsilon} \alpha \wedge \bar{\beta}=I_{0} \log \epsilon+I_{1}(\lambda)+O(\epsilon)
$$

where

$$
I_{0}=2(-1)^{n-1} \int_{D} \operatorname{Res} \alpha \wedge \overline{\operatorname{Res} \beta}
$$

and for $\varphi \in C^{\infty}(X, \mathbb{R})$,

$$
I_{1}\left(e^{\varphi} \lambda\right)=I_{1}(\lambda)-2(-1)^{n-1} \int_{D} \operatorname{Res}_{\partial}(\varphi \alpha) \wedge \overline{\operatorname{Res} \beta}
$$


The integrals over $D$ in this theorem are understood as integrals of representatives in the equivalence classes of the residues: in $I_{0}$ the Leray residues are de Rham cohomology classes (while Res $\beta$ is defined as a closed form) and the integral is independent of choices by Stokes's theorem. In the second integral over $D, \operatorname{Res}_{\partial}$ is defined up to addition of a $\partial$-exact form. Since $\overline{\operatorname{Res} \beta}$ is a closed form of type $(0, n-1)$, we have

$$
\int_{D} \partial \gamma \wedge \overline{\operatorname{Res} \beta}=\int_{D} \mathrm{~d} \gamma \wedge \overline{\operatorname{Res} \beta}=\int_{D} \mathrm{~d}(\gamma \wedge \overline{\operatorname{Res} \beta})=0,
$$

and the integral is well-defined.

The proof of Theorem 3.2 is done by reducing it to a local calculation via a partition of unity. It follows from a local version of the theorem, which we now state, in which we take $\alpha$ to be smooth with compact support.

Let $X$ be a not necessarily compact complex manifold and $D \subset X$ a smooth hypersurface. Let $\Gamma_{c}\left(X, \mathcal{A}_{X}^{p, q}(* D)\right)$ be the vector space of sections of $\mathcal{A}_{X}^{p, q}(* D)$ with compact support. For a cut-off function $\lambda$ and $\epsilon>0$ we consider the sesquilinear pairing

$$
\langle,\rangle_{\lambda, \epsilon}: \Gamma_{C}\left(X, \mathcal{A}_{X}^{n, 0}(* D)\right) \times \Gamma\left(X, \Omega_{X}^{n}(D)\right) \rightarrow \mathbb{C} .
$$

defined by the integral

$$
\langle\alpha, \beta\rangle_{\lambda, \epsilon}=\frac{(-1)^{n-1}}{2 \pi \mathrm{i}} \int_{\lambda \geq \epsilon} \alpha \wedge \bar{\beta}
$$

Theorem 3.3. Let $D \subset X$ be a smooth hypersurface in an arbitrary complex manifold $X$ and $m \in\{0,1,2, \ldots\}$.

(i) Let $\alpha \in \Gamma_{c}\left(X, \mathcal{A}_{X}^{n, 0}(m D)\right)$. Then, as $\epsilon \rightarrow 0$,

$$
\langle\alpha, \beta\rangle_{\lambda, \epsilon}=2 \log \epsilon \int_{D} \operatorname{Res}_{\partial} \alpha \wedge \overline{\operatorname{Res} \beta}+I_{1}(\lambda)+O(\epsilon)
$$

for some function $I_{1}(\lambda)$ of the cut-off function.

(ii) Let $\varphi \in C^{\infty}(X, \mathbb{R})$. Then

$$
I_{1}\left(e^{\varphi} \lambda\right)=I_{1}(\lambda)-2 \int_{D} \operatorname{Res}_{\partial}(\varphi \alpha) \wedge \overline{\operatorname{Res} \beta},
$$

Theorem 3.2 follows from Theorem 3.3 via the embedding $\Omega_{X}^{n}(* D) \hookrightarrow \mathcal{A}^{n, 0}(* D)$. We can replace $\operatorname{Res}_{\partial}$ by the Leray residue Res in the coefficient of $\log \epsilon$ thanks to Proposition 2.6. We prove Theorem 3.3 in Section 5 by a local calculation involving applying the Riemann Mapping Theorem to relate the region $\lambda<\epsilon$ to the region $|f|<\epsilon$ for a local 
equation $f=0$ of $D$. To do this we study the asymptotic behaviour of Riemann mappings in the next section.

Remark 3.4. The integrals $\int_{D} \operatorname{Res}_{\partial} \alpha \wedge \overline{\operatorname{Res} \beta}$ appearing in Theorems 3.2 and 3.3 can be understood in terms of principal value currents, see [4], as suggested to us by the referee. Let $L=O(D)$ be the sheaf of meromorphic sections with at most simple pole on $D$. Then $D$ is given by $s_{D}=0$ for the canonical section $s_{D}$ of $L$ and $s_{D}^{-N}$ canonically defines a principal value de Rham current with values in $L^{-N}$, also denoted $s_{D}^{-N}$ : its value on a test form $\omega$ of degree $(n, n)$ is $\lim _{\epsilon \rightarrow 0} \int_{\left\|s_{D}\right\|>\epsilon} s_{D}^{-N} \omega$ for any choice of Hermitian form \| $\|$. Its $\bar{\partial}$ derivative $\bar{\partial}\left(s_{D}^{-N}\right)$ is supported on $D$ and $\bar{h} \bar{\partial}\left(s_{D}^{-N}\right), \mathrm{d} \bar{h} \wedge \bar{\partial}\left(s_{D}^{-N}\right)$ vanish for any local holomorphic function $h$ vanishing on $D$. Thus for any antiholomorphic $(0, n-1)$-form $\bar{\rho}$ on $D$, such as $\overline{\operatorname{Res} \beta}$, we can define a de Rham current $\bar{\rho} \wedge \bar{\partial}\left(s_{D}^{-N}\right)$ by locally extending $\bar{\rho}$ to an antiholomorphic form on $X$. Since the result is independent of the choice of extension, we get a globally well-defined de Rham current of degree $(0, n)$ with values in $L^{-N}$. Suppose that $\alpha$ is a smooth $(n, 0)$ on $X \backslash D$ such that $\tilde{\alpha}=s_{D}^{N} \alpha$ is a smooth $(n, 0)$-form on $X$ with values in $L^{N}$ and compact support. Then it can be checked that

$$
\int_{D} \operatorname{Res}_{\partial} \alpha \wedge \bar{\rho}=\frac{1}{2 \pi \mathrm{i}}\left\langle\bar{\partial}\left(s_{D}^{-N}\right) \wedge \bar{\rho}, \tilde{\alpha}\right\rangle
$$

the value of the current on the test form $\tilde{\alpha}$.

\section{Asymptotic Riemann Mapping}

Let $\mu(z)=k(z)|z|^{2}$ where $k(z)>0$ is a positive real analytic function in a neighbourhood of $0 \in \mathbb{C}$. For small $\epsilon$ the level sets $\mu^{-1}\left(\epsilon^{2}\right)$ bound a simply connected domain $E_{\epsilon}$ containing 0 and by Riemann's mapping theorem there exists a univalent holomorphic map $h$ from $E_{\epsilon}$ on to the disk $|w|<\epsilon$. The map is unique if we require it to be normalized, namely that $h(0)=0$ and $h^{\prime}(0)>0$. The following results give a description of the behaviour of $h$ as $\epsilon \rightarrow 0$.

Theorem 4.1. Let $\mu(z)=k(z)|z|^{2}$ where $k(z)>0$ is a positive real analytic function in a neighbourhood of $0 \in \mathbb{C}$. Then there exist positive constants $R_{1}=R_{1}(\mu), R_{2}=R_{2}(\mu)$ and a holomorphic function $h(\epsilon, z)$ defined for $|\epsilon|<R_{1},|z|<R_{2}$ such that for $0<\epsilon<R_{1}$, $z \mapsto h(\epsilon, z)$ is the normalized univalent map sending the domain $\mu(z)<\epsilon^{2}$ on to the disk $|w|<\epsilon$. Moreover $h$ is an even function of $\epsilon$. 
Theorem 4.2. The limiting Riemann mapping $h(0, z)$ has the following description. Let $\mu(z)=\sum_{r, s} c_{r, s} z^{r+1} \bar{z}^{s+1}$ be the Taylor expansion of $\mu$. Then

$$
h(0, z)=\sum_{r=0}^{\infty} \frac{C_{r, 0}}{\sqrt{C_{0,0}}} z^{r+1}
$$

Example 4.3. Suppose that $\mu(z)=|h(z)|^{2}$ for some holomorphic function $h(z)$ such that $h(0)=0$ and $h^{\prime}(0)>0$. Then $h(\epsilon, z)=h(z)$ is the normalized univalent map and is independent of $\epsilon$. If $h(z)=z\left(c_{0}+c_{1} z+\cdots\right)$ with $c_{0}>0$, then the Taylor coefficients of $\mu(z)$ are $c_{r, s}=c_{r} \bar{c}_{s}$. In particular $c_{r, 0}=c_{r} c_{0}$ and $c_{0,0}=c_{0}^{2}$.

Example 4.4. Let $\mu(z)=\psi\left(|z|^{2}\right)$ for some real analytic function $\psi$ such that $\psi(0)=$ $0, \psi^{\prime}(0)>0$. Then $h(\epsilon, z)=C(\epsilon) z$ where

$$
C(\epsilon)=\frac{\epsilon}{\sqrt{\psi^{-1}\left(\epsilon^{2}\right)}} .
$$

In this case $c_{0,0}=\psi^{\prime}(0)$ and $c_{r, 0}=0$ for $r>0$, and indeed $h(0, z)=C(0) z=\sqrt{\psi^{\prime}(0)} z$.

Example 4.5. This is rather a counterexample, showing that regularity assumptions on $\mu$ are necessary. Consider the family of maps

$$
W=h(\epsilon, z)=\frac{2 \epsilon z}{2 \epsilon+z}
$$

with parameter $\epsilon>0$. Then $z \mapsto h(\epsilon, z)$ is the univalent map sending $\mu(z)<\epsilon^{2}$ to $|w|<\epsilon$, where $\mu: \mathbb{C} \rightarrow \mathbb{R}_{\geq 0}$ is defined by the condition that $\mu(0)=0$ and $\mu(z)=\epsilon^{2}$ on the circle $|z-2 \epsilon / 3|=4 \epsilon / 3$ (these circles enclose the origin and foliate $\mathbb{C} \backslash 0$ ). It can be shown that $\mu(z)$ is smooth except at $z=0$ where it is continuous and obeys $\mu(z) \leq$ const $|z|^{2}$. The functions of the family $h(\epsilon, z)$ does not have a common domain of definition and do not have a reasonable limit as $\epsilon \rightarrow 0$.

Example 4.6. Another counterexample, showing that Theorem 4.1 can fail if $\mu(z)$ is not of the form $k(z)|z|^{2}$. Let

$$
\mu(x+\mathrm{i} y)=\frac{x^{2}}{a^{2}}+\frac{y^{2}}{b^{2}}, \quad \text { with } a \neq b .
$$

Since $\mu$ is homogeneous the normalized Riemann mapping $h(\epsilon, z)$ sending $\mu(z)<\epsilon^{2}$ to $|w|<\epsilon$ obeys

$$
h(\epsilon, z)=\epsilon h(1, z / \epsilon),
$$


Since the Riemann mapping $h(1, z)$ of the ellipse is non-linear, $h(\epsilon, z)$ does not have a limit as $\epsilon \rightarrow 0$.

Remark 4.7. The assumption of real analyticity of $\mu$ seems to be essential. It implies that the boundary of the level sets for small level are real analytic so that-by the Schwarz reflection principle - the Riemann mappings have an analytic continuation to a neighbourhood of the boundary, a necessary condition for the existence of the limiting map, which is not fulfilled for general smooth $\mu$. As Example 4.5 shows, even the real analyticity of the level sets is not sufficient for the existence of a limiting Riemann mapping.

Remark 4.8. Let $\mu$ be as in Theorem 4.1. Let $\psi$ be a real analytic local diffeomorphism on a neighbourhood of $0 \in \mathbb{R}$ such that $\psi(0)=0, f$ a local biholomorphic map such that $f(0)=0$. Then $\tilde{\mu}=\psi \circ \mu \circ f$ also obeys the assumptions of Theorem 4.1 and the corresponding normalized Riemann mappings are related by

$$
\frac{f^{\prime}(0)}{\left|f^{\prime}(0)\right|} \tilde{h}(\tilde{\epsilon}, z)=h(\epsilon, f(z)), \quad \tilde{\epsilon}^{2}=\psi\left(\epsilon^{2}\right) .
$$

To prove Theorems 4.1 and 4.2 it is convenient to consider, instead of the Riemann mapping, the inverse Riemann mapping, the univalent map $w \mapsto z=f_{\epsilon}(w)=$ $w\left(a_{0}(\epsilon)+a_{1}(\epsilon) w+\cdots\right)$ with $a_{0}(\epsilon)>0$, sending $|w|^{2}<\epsilon^{2}$ on to $\mu(z)<\epsilon^{2}$. Also, by rescaling $\epsilon$ we may assume that $\mu(z)=k(z)|z|^{2}$ with $k(0)=1$.

The proof is based on the contraction principle: we write the equation for the coefficients of the inverse Riemann mapping as a fixed point equation for a map that is a contraction in a suitable metric space and depends smoothly on $\epsilon$ including at $\epsilon=0$.

Lemma 4.9. Let $\mu(z)=\sum_{r, s=0}^{\infty} c_{r, s} z^{r+1} \bar{z}^{s+1}$ be the Taylor expansion of $\mu$. Let $\epsilon>0$ be small. Then $z=\sum_{n=0}^{\infty} a_{n}(\epsilon) W^{n+1}$ is the normalized univalent map sending $D_{\epsilon}=\{w \in \mathbb{C}$ : $|W|<\epsilon\}$ on to $E_{\epsilon}=\left\{z \in \mathbb{C}: \mu(z)<\epsilon^{2}\right\}$ if and only if the series converges to a univalent function on $D_{\epsilon}$ with $a_{0}(\epsilon)>0$ and the sequence $a(\epsilon)=\left(a_{n}(\epsilon)\right)_{n=0}^{\infty}$ obeys

$$
a_{n}(\epsilon)=F_{n}(\epsilon ; a(\epsilon)), \quad n=0,1,2, \ldots,
$$

where for $n \geq 1$,

$$
\begin{aligned}
F_{n}(\epsilon ; a)= & -\sum_{r+s>0} c_{r, s} \sum_{r+|p|-s-|q|=n} \epsilon^{2 s+2|q|} a_{p_{1}} \cdots a_{p_{r+1}} \bar{a}_{q_{1}} \cdots \bar{a}_{q_{s+1}} \\
& -a_{n}\left(\bar{a}_{0}-1\right)-\sum_{k \geq 1} a_{n+k} \bar{a}_{k} \epsilon^{2 k}
\end{aligned}
$$


with $|p|=\sum_{i=1}^{r} p_{i}$ and $|q|=\sum_{i=1}^{s} q_{i}$, and for $n=0$,

$$
\begin{aligned}
F_{0}(\epsilon ; a)=1 & -\frac{1}{2} \sum_{r+s>0} c_{r, s} \sum_{r+|p|-s-|q|=0} \epsilon^{2 s+2|q|} a_{p_{1}} \cdots a_{p_{r+1}} \bar{a}_{q_{1}} \cdots \bar{a}_{q_{s+1}} \\
& -\frac{1}{2}\left|a_{0}-1\right|^{2}-\frac{1}{2} \sum_{k \geq 1} a_{k} \bar{a}_{k} \epsilon^{2 k} .
\end{aligned}
$$

Proof. Since the curve $\mu(z)=\epsilon^{2}$ is smooth if $\epsilon$ is small enough, the Riemann mapping and its inverse extend to smooth maps on the closure (in fact to holomorphic maps on a neighbourhood of the closure with our assumption of real analyticity of $\mu$ ). Thus the restriction to the boundary of the inverse Riemann mapping $f_{\epsilon}(w)$ has a convergent Fourier series and sends the circle $|w|=\epsilon$ to the curve $\mu(z)=\epsilon^{2}$.

We write this condition as a fixed point equation. Write $f_{\epsilon}(w)=w\left(1+g_{\epsilon}(w)\right)$, $k(z)=1+m(z)$, so that

$$
m(z)=\sum_{r+s>0} c_{r, s} z^{r} \bar{z}^{s}
$$

Then the equation for $g_{\epsilon}(w)=a_{0}-1+\sum_{n=1}^{\infty} a_{n} W^{n}$ is

$$
\left(1+m\left(f_{\epsilon}(\epsilon u)\right)\right)\left|1+g_{\epsilon}(\epsilon u)\right|^{2}=1, \quad|u|=1 .
$$

We can write this equation in the form

$$
g_{\epsilon}(\epsilon u)+\bar{g}_{\epsilon}\left(\epsilon u^{-1}\right)=-m\left(f_{\epsilon}(\epsilon u)\right)\left|1+g_{\epsilon}(\epsilon u)\right|^{2}-\left|g_{\epsilon}(\epsilon u)\right|^{2}, \quad|u|=1 .
$$

Being an identity of real-valued Fourier series, (4) holds if and only if the coefficients of $u^{n}$ for $n \geq 0$ coincide on both sides. Let $[\varphi(u)]_{n}$ denote the $n$th Fourier coefficient of a smooth function $\varphi(u)$ on the unit circle $|u|=1$. Together with the condition that $a_{0}$ is real and positive, we get the equivalent condition

$$
\begin{aligned}
& a_{0}=1-\frac{1}{2}\left[m\left(f_{\epsilon}(\epsilon u)\right)\left|1+g_{\epsilon}(\epsilon u)\right|^{2}+\left|g_{\epsilon}(\epsilon u)\right|^{2}\right]_{0}, \\
& a_{n}=-\frac{1}{\epsilon^{n}}\left[m\left(f_{\epsilon}(\epsilon u)\right)\left|1+g_{\epsilon}(\epsilon u)\right|^{2}+\left|g_{\epsilon}(\epsilon u)\right|^{2}\right]_{n}, \quad n \geq 1 .
\end{aligned}
$$

This equation has the form $a_{n}=F_{n}\left(\epsilon ; a_{0}, a_{1}, a_{2}, \ldots\right)$, with

$$
\begin{aligned}
F_{n}(\epsilon ; a)= & -\sum_{r+s>0} c_{r, s} \sum_{r+|p|-s-|q|=n} \epsilon^{r+s+|p|+|q|-n} a_{p_{1}} \cdots a_{p_{r+1}} \bar{a}_{q_{1}} \cdots \bar{a}_{q_{s+1}} \\
& -a_{n}\left(\bar{a}_{0}-1\right)-\sum_{k \geq 1} a_{n+k} \bar{a}_{k} \epsilon^{2 k}
\end{aligned}
$$




$$
\begin{aligned}
= & -\sum_{r+s>0} c_{r, s} \sum_{r+|p|-s-|q|=n} \epsilon^{2 s+2|q|} a_{p_{1}} \cdots a_{p_{r+1}} \bar{a}_{q_{1}} \cdots \bar{a}_{q_{s+1}} \\
& -a_{n}\left(\bar{a}_{0}-1\right)-\sum_{k \geq 1} a_{n+k} \bar{a}_{k} \epsilon^{2 k}
\end{aligned}
$$

for $n \geq 1$. For $n=0$ the calculation is similar.

We notice that $F(\epsilon ; a)=\left(F_{n}(\epsilon ; a)_{n=0}^{\infty}\right)$ is defined (a priori only as a formal power series in the $a_{j}$ ) also for $\epsilon=0$. In this case we can find the answer explicitly.

Lemma 4.10. Let $\mu(z)=\sum_{r, s=0}^{\infty} c_{r, s} z^{r+1} \bar{z}^{s+1}$ with $c_{0,0}=1$. Let

$$
h(z)=\sum_{r=0}^{\infty} c_{r, 0} z^{r+1}
$$

and $h^{-1}(w)=w\left(a_{0}+a_{1} w+\cdots\right)$ its inverse. Then $a=\left(a_{n}\right)_{n=0}^{\infty}$ is a solution of $a=$ $F(0 ; a)$.

Proof. By Example 4.3, if $\mu(z)=|h(z)|^{2}$ for some holomorphic function $h$ such that $h(0)=0, h^{\prime}(0)=1$ then $f_{\epsilon}=h^{-1}$ is the univalent map of Lemma 4.9 and is independent of $\epsilon$. In this case the Taylor coefficients $c_{r, 0}$ are precisely the Taylor coefficients of $h$. Thus the equation $a=F(0 ; a)$, which only involves $c_{r, s}$ with $s=0$, is the same for a function $\mu$ with arbitrary Taylor coefficients $c_{r, s}$ as for $\mu=|h(z)|^{2}$ with $h(z)=\sum c_{r, 0} z^{r+1}$.

Let $\ell_{R}^{1}(\mathbb{C})$ be the Banach space of sequences $a=\left(a_{n}\right)_{n=0}^{\infty}$ of complex numbers with finite weighted $\ell^{1}$-norm $\|a\|_{R}=\sum_{n=0}^{\infty}\left|a_{n}\right| R^{n}$.

Lemma 4.11. Let $0<\delta<1$. With the same notations and assumptions as in Lemma 4.9 , there exists an $R>0$ depending on $\mu$ and $\delta$, such that the power series $F$ defines a holomorphic map

$$
\{\epsilon \in \mathbb{C}:|\epsilon|<R\} \times B_{\delta}\left(a^{\circ}\right) \rightarrow B_{\delta}\left(a^{\circ}\right),
$$

where

$$
B_{\delta}\left(a^{\circ}\right)=B_{\delta}\left(a^{\circ} ;\|\|_{R}\right)=\left\{a \in \ell_{R}^{1}(\mathbb{C}):\left\|a-a^{\circ}\right\|_{R}<\delta\right\}
$$

is the open ball of radius $\delta$ centred at $a^{\circ}=(1,0,0, \ldots)$ in $\ell_{R}^{1}(\mathbb{C})$. 
Proof. We show that $F(\epsilon, a)-a^{\circ}$ is given by a power series in $\epsilon, a_{0}-1, a_{n}, \bar{a}_{n}$ which converges for $|\epsilon|<R,\left\|a-a^{\circ}\right\|_{R}<\delta$, namely that the series

$$
\left\|F(\epsilon ; a)-a^{\circ}\right\|_{R}=\left|F_{0}(\epsilon ; a)-1\right|+\sum_{n=1}^{\infty}\left|F_{n}(\epsilon ; a)\right| R^{n} .
$$

converges absolutely in the specified domain.

Let $F(\epsilon ; a)-a^{\circ}=A+B$ where $A$ is the part involving $c_{r, s}$ and $B$ is the quadratic, $C_{r, s}$-independent part. In $\left|F_{0}(\epsilon ; a)-1\right|$ we use that $1 / 2 \leq 1$ to obtain a single summation for $A$ :

$$
\begin{aligned}
\|A\|_{R} & \leq \sum_{r+s>0}\left|c_{r, s}\right| \sum_{p, q}|\epsilon|^{2 s+2|q|} R^{r+|p|-s-|q|}\left|a_{p_{1}} \cdots a_{p_{r+1}} \bar{a}_{q_{1}} \cdots \bar{a}_{q_{s+1}}\right| \\
& \leq \sum_{r+s>0}\left|c_{r, s}\right| R^{r}\left(|\epsilon|^{2} / R\right)^{s}\|a\|_{R}^{r+1}\|a\|_{\epsilon^{2} / R}^{s+1} \\
& \leq \sum_{r+s>0}\left|c_{r, s}\right| R^{r+s}\|a\|_{R}^{r+s+2} .
\end{aligned}
$$

In the last inequality we use $|\epsilon| \leq R$ so that \|\|$_{\epsilon^{2} / R} \leq\|\|_{R}$. To estimate $B$ we set $a=a^{\circ}+b$ :

$$
\begin{aligned}
\|B\|_{R} & \leq \frac{1}{2} \sum_{k=0}^{\infty}\left|b_{k}\right|^{2}|\epsilon|^{2 k}+\sum_{n=1}^{\infty} \sum_{k=0}^{\infty}\left|b_{n+k}\right|\left|b_{k}\right| R^{n}|\epsilon|^{2 k} \\
& \leq \frac{1}{2} \sum_{k=0}^{\infty}\left|b_{k}\right|^{2} R^{2 k}+\sum_{n=1}^{\infty} \sum_{k=0}^{\infty}\left|b_{n+k}\right| R^{n+k}\left|b_{k}\right| R^{k} \\
& =\frac{1}{2} \sum_{k=0}^{\infty}\left|b_{k}\right|^{2} R^{2 k}+\frac{1}{2} \sum_{k \neq n}\left|b_{n}\right| R^{n}\left|b_{k}\right| R^{k} \\
& \leq \frac{1}{2}\|b\|_{R}^{2}
\end{aligned}
$$

Therefore, we have the estimate

$$
\left\|F(\epsilon ; a)-a^{\circ}\right\| \leq \sum_{r+s>0}\left|c_{r, s}\right| R^{r+s}\|a\|_{R}^{r+s+2}+\frac{1}{2}\left\|a-a^{\circ}\right\|_{R}^{2} .
$$

Assume that $\left\|a-a^{\circ}\right\|_{R}<\delta$. Then $\|a\|_{R} \leq\left\|a^{\circ}\right\|_{R}+\delta=1+\delta$ and

$$
\left\|F(\epsilon ; a)-a^{\circ}\right\|<\sum_{r+s>0}\left|c_{r, s}\right| R^{r+s}(1+\delta)^{r+s+2}+\delta^{2} / 2 .
$$

Now choose $R$ so small that

$$
\sum_{r+s>0}\left|C_{r, s}\right| R^{r+s}(1+\delta)^{r+s+2} \leq \delta-\delta^{2} / 2
$$

Then $\left\|F(a)-a^{\circ}\right\|_{R}<\delta$. 
Lemma 4.12. With the notation of the previous lemma, $R$ can be chosen so that $a \mapsto$ $F(\epsilon ; a)$ is a contraction of $B_{\delta}\left(a^{\circ}\right) \subset \ell_{R}^{1}(\mathbb{C})$ for all $\epsilon \in \mathbb{C}$ such that $|\epsilon|<R$.

Proof. It is sufficient to show that the differential has norm $<1$. Since $F$ is holomorphic we can compute the differential term by term. The result is that the differential

$$
d_{a} F(\epsilon ; a)(h)=\lim _{t \rightarrow 0} \frac{1}{t}(F(\epsilon ; a+t h)-F(\epsilon ; a))
$$

is obtained by replacing in each monomial of the power series defining $F$ one occurrence of $a_{p}$ or $\bar{a}_{p}$ by $h_{p}$ or $\bar{h}_{p}$ and multiplying by the degree. We can then estimate the norm in the same way as in the previous lemma. For $h \in \ell_{R}^{1}(\mathbb{C})$,

$$
\begin{aligned}
\left\|d_{a} F_{n}(\epsilon ; a)(h)\right\|_{R} & \leq \sum_{r+s>0}(r+s+2)\left|c_{r, s}\right| R^{r+s}\|a\|_{R}^{r+s+1}\|h\|_{R} \\
& +\|h\|_{R}\left\|a-a^{\circ}\right\|_{R} \\
& \leq \sum_{r+s>0}(r+s+2)\left|c_{r, s}\right| R^{r+s}(1+\delta)^{r+s+1}\|h\|_{R} \\
& +\delta\|h\|_{R}
\end{aligned}
$$

Since $\mu$ is real analytic, for any given $\delta \in(0,1)$ it is possible to choose $R>0$ such that

$$
\sum_{r+s>0}(r+s+2)\left|c_{r, s}\right| R^{r+s}(1+\delta)^{r+s+1} \leq \theta-\delta,
$$

with $\delta<\theta<1$. With this choice of $R,\left\|d_{a} F(\epsilon ; a)\right\|_{R} \leq \theta$.

Proof of Theorems 4.1 and 4.2. By the Banach contraction principle there is a unique sequence $a(\epsilon) \in B_{\delta}\left(a^{\circ}\right)$ obeying the fixed point equation $a(\epsilon)=F(\epsilon ; a(\epsilon))$. Since $\sum_{n=0}^{\infty}\left|a_{n}(\epsilon)\right| R^{n}<\infty$ and $\left|a_{0}(\epsilon)-1\right| \leq\left\|a(\epsilon)-a^{\circ}\right\|_{R}<\delta<1$, the power series $f_{\epsilon}(w)=$ $w\left(a_{0}(\epsilon)+a_{1}(\epsilon) w+\cdots\right)$ is absolutely convergent for $|w|<R$ and defines a univalent map for $|\epsilon|<R_{1}$ for sufficiently small $R_{1}\left(R_{1}=R / 2\right.$ will do). Also $a_{0}(\epsilon)$ is real for real $\epsilon$ since $F_{0}$ is real; because $\left|a_{0}(\epsilon)-1\right|<1$, it is positive and thus $f_{\epsilon}$ is normalized. By Lemma $4.9 f_{\epsilon}(W)$ is the required univalent map. By Lemma $4.11 F$ is holomorphic, and by Lemma 4.12 the differential Id $-d_{a} F(\epsilon ; a(\epsilon))$ at $a(\epsilon)$ of the map $a \mapsto a-F(\epsilon ; a)$ is an automorphism of the Banach space $\ell_{R}^{1}(\mathbb{C})$. By the implicit function theorem for analytic maps in Banach spaces, see [3, Chapter 1, Theorem 174] $a(\epsilon)$ is an analytic function of $\epsilon$ and thus $f_{\epsilon}(w)$ is analytic as a function of $\epsilon$ and $w$. It follows that the Riemann mapping $h(\epsilon, z)=f_{\epsilon}^{-1}(z)$ is also holomorphic. The fact that $h$ is an even function of $\epsilon$ follows most 
simply from the invariance of the fixed point equation in Lemma 4.9 under $\epsilon \rightarrow-\epsilon$. This proves Theorem 4.1. Theorem 4.2 then follows from Lemma 4.10.

\section{Proof of Theorem 3.3}

By using a partition of unity we may assume that $X$ is an open neighbourhood of $0 \in \mathbb{C}^{n}$ and that $D$ is defined by the equation $z_{1}=0$ where $z_{1}$ is the first coordinate function on $\mathbb{C}^{n}$. The main technical step is to settle the case where $\alpha=\partial \gamma$ is exact:

Lemma 5.1. Let $m \in\{0,1,2, \ldots\}$. If $\alpha=\partial \gamma$ with $\gamma \in \Gamma_{c}\left(X, \mathcal{A}_{X}^{n-1,0}(m D)\right)$ then, as $\epsilon \rightarrow 0$,

$$
\langle\alpha, \beta\rangle_{\lambda, \epsilon}=2 \int_{D} \operatorname{Res}_{\partial}(\partial \log \lambda \wedge \gamma) \wedge \overline{\operatorname{Res} \beta}+O(\epsilon)
$$

Proof. We first reduce the general case to the case where $\lambda^{2}$ is real analytic, and in fact a polynomial. Let $\lambda$ be a cut-off function and $\lambda_{N}$ be the cut-off function such that $\lambda_{N}^{2}$ is the degree $N$ Taylor polynomial in the variable $z_{1}$ at $z_{1}=0$. We show that for fixed $\alpha, \beta$, and $N$ large enough,

$$
\langle\alpha, \beta\rangle_{\lambda, \epsilon}=\langle\alpha, \beta\rangle_{\lambda_{N}, \epsilon}+O(\epsilon)
$$

For small $\epsilon, U_{\epsilon}=\lambda^{-1}([0, \epsilon)) \cap \operatorname{supp} \alpha$ is contained in the region $\left|z_{1}\right|<C_{1} \epsilon$ for some $C_{1}>0$ and thus $\left|\lambda_{N}(z)-\lambda(z)\right|<C_{2} \epsilon^{N+1}$ for all $z \in U_{\epsilon}$ and some $C_{2}>0$. It follows that the level set $\lambda_{N}^{-1}(\epsilon)$ is contained in $\lambda^{-1}\left(\left[\epsilon-C \epsilon^{N+1}, \epsilon+C \epsilon^{N+1}\right]\right.$. The difference between the integrals over $\lambda(z)>\epsilon$ and $\lambda_{N}(z)>0$ is then estimated by the volume of the region, which is less than const $\epsilon^{N}$ times the maximum of the integrand, which is less than const $\epsilon^{-m-1}$ if $\alpha$ has a pole of order $m$. For $N>m-1$ the difference vanishes in the limit $\epsilon \rightarrow 0$.

So we assume from now on that $\lambda^{2}$ is a real analytic function of $z$.

By Stokes' theorem,

$$
\langle\partial \gamma, \beta\rangle_{\lambda, \epsilon}=\frac{(-1)^{n}}{2 \pi i} \int_{\lambda=\epsilon} \gamma \wedge \bar{\beta}
$$

We prove the identity in several steps.

(a) The one-dimensional case with $\lambda(z)=|z|$.

Suppose $n=1$ and write $z_{1}=z$. Then $\gamma(z)=z^{-m} \sigma(z)$ for some smooth function $\sigma$ and $\bar{\beta}=\bar{b}(\bar{z}) \mathrm{d} \bar{z} / \bar{z}$ for some holomorphic function $\bar{b}$ defined around 0 . Let us first assume that 
$\lambda(z)=|z|$ and let $\sigma(z) \sim \sum_{r, s=0}^{\infty} \sigma_{r s} z^{r} \bar{z}^{s}$ be the Taylor series of $\sigma$. Then

$$
\int_{|z|=\epsilon} \gamma \wedge \bar{\beta}=-\sum_{r+s \leq N} \sigma_{r, s} \epsilon^{r+s-m} \int_{|u|=1} u^{r-s-m} \bar{b}\left(\epsilon u^{-1}\right) \frac{\mathrm{d} u}{u}+O(\epsilon)
$$

for $N$ sufficiently large, say $N \geq m+1$. Thus we have to evaluate

$$
\epsilon^{r+s-m} \int_{|u|=1} u^{r-s-m}\left(\epsilon u^{-1}\right)^{l} \frac{\mathrm{d} u}{u}=2 \pi i \delta_{r, s+m+l} \epsilon^{2 s+2 l},
$$

for $r, s, m, l \geq 0$. Thus only one term in the sum and only the leading term $\bar{b}(0)$ survive the limit $\epsilon \rightarrow 0$. Therefore

$$
\int_{|z|=\epsilon} \gamma \wedge \bar{\beta}=-2 \pi i \sigma_{m, 0} \bar{b}(0)+O(\epsilon)
$$

On the other hand, $b(0)=\operatorname{Res} \beta$ and

$$
\operatorname{Res}_{\partial}(\partial \log \lambda \gamma)=\operatorname{Res}_{\partial} \frac{\mathrm{d} z}{Z} \gamma(z)=\sigma_{m, 0},
$$

as computed in Example 2.5. The proof is complete.

\section{(b) The one-dimensional case with general $\lambda$.}

Let again $n=1$, but with a general cut-off function $\lambda$. By Theorem 4.1, for sufficiently small $\epsilon$, the normalized biholomorphic Riemann map $z=g_{\epsilon}(w)$ sending the disk $|w|<\epsilon$ to the domain $\lambda(z)<\epsilon$ extends to a smooth parametrization of the boundary and has an analytic continuation to a holomorphic function of $\epsilon, z$ defined on a polydisk around zero. We change variables to reduce to case (a):

$$
\int_{\lambda(z)=\epsilon} \gamma \wedge \bar{\beta}=\int_{|w|=\epsilon} g_{\epsilon}^{*} \gamma \wedge \overline{g_{\epsilon}^{*} \beta} .
$$

In this formula we may replace $g_{\epsilon}$ by its limit for $\epsilon=0$, since the difference is $\epsilon$ times an integral of the form considered in (a) which has a finite limit as $\epsilon \rightarrow 0$. The required identity

$$
\frac{-1}{2 \pi \mathrm{i}} \int_{\lambda(z)=\epsilon} \gamma \wedge \bar{\beta}=\operatorname{Res}_{\partial}(\partial \log \lambda \gamma) \wedge \overline{\operatorname{Res} \beta}+O(\epsilon)
$$

thus holds in the coordinate $w=g_{0}(z)$ and thus in any coordinate system. 
(c) The case of arbitrary dimension I

Now let $n$ be arbitrary and suppose that

$$
\gamma=f d z_{2} \wedge \cdots \wedge d z_{n}
$$

for some function $f$. Then the integration over each fibre of the projection $z \rightarrow\left(z_{2}, \ldots, z_{n}\right)$ is of the form considered in (b). The claim then follows from (b), (the sign comes from permuting $\mathrm{d} \bar{z}_{1}$ through $\mathrm{d} z_{2} \wedge \cdots \wedge \mathrm{d} z_{n}$.)

\section{(d) The case of arbitrary dimension II}

Now we assume that

$$
\gamma=\frac{\mathrm{d} z_{1}}{z_{1}} \wedge \sigma
$$

for some smooth $(n-2)$-form $\sigma$. On the cycle $\lambda=\epsilon$ we have the identity

$$
\partial \log \lambda+\bar{\partial} \log \lambda=0
$$

Let us again introduce coordinates $z_{1}, z_{2}, \ldots, z_{n}$ and decompose the differential as $\partial=$ $\mathrm{d} z_{1} \partial_{z_{1}}+\partial^{\prime}$. The identity becomes

$$
\mathrm{d} z_{1} \partial_{z_{1}} \log \lambda+\partial^{\prime} \log \lambda \equiv 0 \bmod \left\langle\mathrm{d} z_{1}, \ldots, \mathrm{d} \bar{z}_{n}\right\rangle
$$

modulo the submodule generated by $\mathrm{d} \bar{z}_{i}$, which doesn't contribute when we multiply by the $(0, n)$-form $\bar{\beta}$. Thus

$$
\int_{\lambda=\epsilon} \gamma \wedge \bar{\beta}=-\int_{\lambda=\epsilon} \frac{\partial^{\prime} \log \lambda}{z_{1} \partial_{z_{1}} \log \lambda} \wedge \sigma \wedge \bar{\beta} .
$$

The denominator $z_{1} \partial_{z_{1}} \log \lambda$ is actually smooth and non-zero on $D$, since it can be written as $1+z_{1} \partial_{z_{1}} \log h$, where $\lambda=h\left|z_{1}\right|$ with smooth non-zero $h$.

Now the integral is of the form considered in (c) for which we have proved the claim. Thus

$$
\frac{(-1)^{n}}{2 \pi i} \int_{\lambda=\epsilon} \gamma \wedge \bar{\beta}=-\int_{D} \operatorname{Res}_{\partial}\left(\partial \log \lambda \wedge \frac{\partial^{\prime} \log \lambda}{z_{1} \partial_{z_{1}} \log \lambda} \wedge \sigma\right) \wedge \overline{\operatorname{Res} \beta}+O(\epsilon) .
$$

Since $\partial^{\prime} \log \lambda \wedge \sigma$ is an $(n-1,0)$-form not involving $\mathrm{d} z_{1}$, we may replace $\partial \log \lambda$ by $\mathrm{d} z_{1} \partial_{z_{1}} \log \lambda$ on the right-hand side and we obtain

$$
\frac{(-1)^{n}}{2 \pi i} \int_{\lambda=\epsilon} \gamma \wedge \bar{\beta}=-\int_{D} \operatorname{Res}_{\partial}\left(\frac{\mathrm{d} z_{1}}{z_{1}} \wedge \partial^{\prime} \log \lambda \wedge \sigma\right) \wedge \overline{\operatorname{Res} \beta}+O(\epsilon)
$$




$$
\begin{aligned}
& =-\int_{D} \operatorname{Res}_{\partial}\left(\frac{\mathrm{d} z_{1}}{z_{1}} \wedge \partial \log \lambda \wedge \sigma\right) \wedge \overline{\operatorname{Res} \beta}+O(\epsilon) \\
& =\int_{D} \operatorname{Res}_{\partial}(\partial \log \lambda \wedge \gamma) \wedge \overline{\operatorname{Res} \beta}+O(\epsilon)
\end{aligned}
$$

so that the claim also holds in this case.

\section{(e) The case of arbitrary dimension III}

We finally deal with the most general case. The form $\gamma$ may be written as

$$
\gamma=f \mathrm{~d} z_{2} \wedge \cdots \wedge \mathrm{d} z_{n}+\mathrm{d} z_{1} \wedge \sigma
$$

for some function $f$ and an $(n-2)$-form $\sigma$ such that $z_{1}^{m} f$ and $z_{1}^{m} \sigma$ are smooth. Since we can add a $\partial$-closed form to $\gamma$ without changing either side of the claim, we may by Proposition 2.1 assume that $\sigma$ has a first order pole. Indeed if $\sigma$ has a pole of order $p+1>1$, say $\sigma=z_{1}^{-p-1} \tau$, then $\mathrm{d} z_{1} \wedge \sigma=\frac{1}{p} z_{1}^{-p} \partial \tau-\partial\left(\frac{1}{p} z_{1}^{-p} \tau\right)$. Thus we can lower the order of the pole by adding exact forms until $\sigma$ has a pole of first order. In this way, the general case is reduced to the cases (c) and (d).

Proof of Theorem 3.3. (i) By Lemma 5.1 and Proposition 2.1, it is sufficient to consider the case where $\alpha$ has a simple pole. Write

$$
\alpha=f \frac{\mathrm{d} z_{1}}{z_{1}} \wedge \mathrm{d} z_{2} \wedge \cdots \wedge \mathrm{d} z_{n}, \quad \beta=g \frac{\mathrm{d} z_{1}}{z_{1}} \wedge \mathrm{d} z_{2} \wedge \cdots \wedge \mathrm{d} z_{n},
$$

where $f$ is a function with compact support and $g$ is holomorphic. We may assume that the support of $f$ is contained in the region $\left|z_{1}\right|<1$. In the integral of $\alpha \wedge \bar{\beta}$ we may replace $f$ and $\bar{g}$ by their values at $z_{1}=0$, as the difference is a regularized absolutely convergent integral, whose limit as $\epsilon \rightarrow 0$ is independent of the choice of $\lambda$. We obtain

$$
\int_{\left|z_{1}\right| \leq 1, \lambda \geq \epsilon} \alpha \wedge \bar{\beta}=(-1)^{n-1} \int_{D} \int_{\left|z_{1}\right| \leq 1, \lambda \geq \epsilon} \frac{\mathrm{d} z_{1} \wedge \mathrm{d} \bar{z}_{1}}{z_{1} \bar{z}_{1}} \wedge \operatorname{Res} \alpha \wedge \overline{\operatorname{Res} \beta}+\cdots,
$$

where the dots denote a term whose limit as $\epsilon \rightarrow 0$ exists and is independent of $\lambda$. We are left with a two-dimensional integral over $z_{1}$ which we evaluate in polar coordinates: let $r=r_{\epsilon}(\theta)=\epsilon / h(0)(1+O(\epsilon))$ the polar parametrization of the curve $\lambda\left(z_{1}\right)=\epsilon$ with $\lambda\left(z_{1}\right)=\left|z_{1}\right| h\left(z_{1}\right)$. Then

$$
\int_{\left|z_{1}\right| \leq 1, \lambda \geq \epsilon} \frac{\mathrm{d} z_{1} \wedge \mathrm{d} \bar{z}_{1}}{z_{1} \bar{z}_{1}}=-2 i \int_{0}^{2 \pi} \int_{r_{\epsilon}(\theta)}^{1} \frac{\mathrm{d} r}{r} \mathrm{~d} \theta
$$




$$
\begin{aligned}
& =2 i \int_{0}^{2 \pi} \log r_{\epsilon}(\theta) \mathrm{d} \theta \\
& =4 \pi i \log (\epsilon / h(0))+O(\epsilon) .
\end{aligned}
$$

It follows that

$$
\langle\alpha, \beta\rangle_{\lambda, \epsilon}=2 \int_{D} \log \left(\frac{\epsilon}{\left.h\right|_{D}}\right) \operatorname{Res} \alpha \wedge \overline{\operatorname{Res} \beta}+\cdots, \quad \lambda=h\left|z_{1}\right|,
$$

up to a finite term whose limit as $\epsilon \rightarrow 0$ is independent of $\lambda$. Since Res coincides with $\operatorname{Res}_{\partial}$ for $(n, 0)$-forms with simple pole, the proof is complete.

(ii) If $\alpha$ has a first order pole, the claim follows from (5): if we multiply $\lambda$ by $\exp \varphi$, then $\left.\log h\right|_{D}$ changes by $\left.\varphi\right|_{D}$, and $\varphi \operatorname{Res}_{\partial} \alpha=\operatorname{Res}_{\partial}(\varphi \alpha)$ in the case of first order pole. By Proposition 2.1, a general form $\alpha$ is the sum of a $\partial$-exact form and a logarithmic form. It is thus sufficient to check the claim when $\alpha=\partial \gamma$ is $\partial$-exact, for which we use Lemma 5.1. Since $\operatorname{Res}_{\partial}$ vanishes on $\operatorname{Im} \partial$, we have

$$
\begin{aligned}
\langle\partial \gamma, \beta\rangle_{e^{\varphi}, \epsilon}-\langle\partial \gamma, \beta\rangle_{\lambda, \epsilon} & =2 \int_{D} \operatorname{Res}_{\partial}(\partial \varphi \wedge \gamma) \wedge \overline{\operatorname{Res} \beta}+O(\epsilon) \\
& =-2 \int_{D} \operatorname{Res}_{\partial}(\varphi \partial \gamma) \wedge \overline{\operatorname{Res} \beta}+O(\epsilon)
\end{aligned}
$$

Thus the claim also holds for $\partial$-exact $\alpha$, completing the proof.

\section{Funding}

This work of G.F. was supported in part by the National Centre of Competence in Research "SwissMAP—The Mathematics of Physics" of the Swiss National Science Foundation.

\section{Acknowledgments}

We thank Curtis McMullen for discussion and suggestions on the topic of Section 4. We thank the referee for his/her corrections, suggestions of improvement and interesting comments.

\section{References}

[1] Deligne, P. “Théorie de Hodge : II." Publications mathématiques de l'IHÉS 40 (1971): 5-57.

[2] Griffiths, P. A. “On the periods of certain rational integrals, I." Annals of Mathematics 90, no. 3 (1969): 460-95.

[3] Hájek, P., and M. Johanis. Smooth Analysis in Banach Spaces, xvi+497 pp. De Gruyter Series in Nonlinear Analysis and Applications, 19. Berlin, De Gruyter, 2014. 
5918 G. Felder and D. Kazhdan

[4] Herrera, M. E. M., and D. I. Lieberman. "Residues and principal values on complex spaces." Mathematische Annalen 194 (1971): 259-94.

[5] Leray, J. “Le calcul différentiel et intégral sur une variété analytique complexe. (Problème de Cauchy. III.)." Bulletin de la Société Mathématique de France 87 (1959): 81-180.

[6] Witten, E. String perturbation theory revisited. arXiv:1209.5461 [hep-th] . 